\title{
LES MECANISMES DE PROTECTION DE LA LIBRE CONCURRENCE EN R.D.C ET LEUR EFFECTIVITE : EXEMPLE DU SECTEUR DES TELECOMMUNICATIONS
}

\author{
Par Pierre DIMANDJA KALONDA et Albert KYEMBE MULUMBWA*
}

\section{INTRODUCTION}

La liberté du commerce et de l'Industrie a été consacrée vers le début même de la période coloniale; et il faut souligner l'importance de cette admission du principe de la liberté commerciale par les puissances colonisatrices de l'époque : on sait qu'à l'époque où nous nous situons, les pays d'Europe n'avaient que faire de la consécration de cette liberté de commerce et de l'industrie. Car chez eux et à l'époque là, l'ordre économique, considéré comme alors désirable, était spontané, résultat du libre jeu des droits individuels et la vie économique échappait à l'action des pouvoirs public. ${ }^{1}$ Alors cette liberté dont il est question était consacrée par l'article premier de l'acte Général de Berlin du 26 Février 1985 qui énonce nettement que «le Commerce des toutes les nations signataire jouira d'une complète liberté dans les limites du bassin géographique du Congo. ${ }^{2}$ On peut du reste ici reproduire des extraits de l'acte Général de Berlin en ce qu'il revient sur le principe consacré de la liberté commerciale : voulant régler, dans un esprit de bonne entente mutuelle, les conditions favorables au développement du commerce et de civilisation de certaine régions de l'Afrique.

Cette disposition susvisée renchérie en disant : « le commerce de toutes les nations jouira d'une complète liberté ». Nous lisons ce qui est toujours à ce propos dans le discours inaugural que prononça le Prince BUMARCK : «l'idée fondamentale de ce programme est de faciliter à toutes les nations commerciales l'accès à l'intérieur de l'Afrique. Le programme de la Conférence ne porte que sur la liberté des commerces dans le bassin du Congo et ses embouchures. Toute puissance qui exercera des droits de souveraineté dans cette région y donnera libre accès à tous les pavillons, sans distinction. Elle ne pourra y concéder de monopoles, ni introduire un traitement différentiel ${ }^{3}$.

La liberté du commerce et de l'industrie qui a été ensuite reprise quant à son esprit par l'article 5 de l'acte de Berlin qui précisa que « toute puissance qui exercera des droits de

\footnotetext{
* Assistants à l'Université de LUBUMBASHI.

1 LUKOMBE NGHENDA : Droit congolais des sociétés Tome I, PUC, 1999, P.229.

2 L'Article 1 de l'acte Général de Berlin du 26/02/1982.

3 Déclaration de la conférence de Berlin. Mouvement géographique, 1892, P.91).
} 
souveraineté dans les territoires susvisés ne pourra y concéder ni monopole d'aucune espèce en matière commerciale $»^{4}$.

Il reste cependant établi que cette liberté du commerce et de l'industrie a été consacrée à nouveau par la convention de Saint Germain-en-Laye signée le 10 Septembre 1912 et laquelle convention a abrogé l'acte général de Berlin et celui de Bruxelles. L'article 4 de la convention de Saint Germain qui repose le principe de l'interdiction des monopoles et privilèges édicté par l'article 5 de l'acte général de Berlin, précisa que « chaque Etat conserve le droit de disposer de ses biens et d'accorder des concessions pour l'exploitation des richesses naturelles du territoire, mais toute règlementation ne pourra comporter aucun traitement différentiel entre les ressortissants des puissances signataires et des Etats membres de la sociétés des nations qui adhéreront à la présente convention $\gg .5$

Le principe général en matière de commerce et d'industrie est celui de la liberté, ce qui implique le droit pour tout commerçant, opérateur économique d'exercer la même activité que d'autres et d'essayer d'attirer la clientèle. Cette liberté peut être tempérée par l'existence des clauses de non concurrence insérées dans certains contrats où l'une des parties s'engageant à ne pas faire concurrence. ${ }^{6}$

Il est important de signaler ici qu'après l'acquisition de l'indépendance le 30 Juin 1960, le Congo ne s'était pas nettement prononcé sur la consécration dans son nouvel ordre juridique, du principe de la liberté du commerce et de l'industrie. La loi fondamentale du 17 Juin 1960 relative aux libertés publiques n'en fait pas mention expresse. Les acteurs trouveraient cependant qu'une allusion aurait faite à l'article 16 de cette loi fondamentale qui précisa que « toute personne à droit à la liberté de réunion pacifique et à la liberté d'association $»{ }^{7}$

Il résulte de la lecture de cette disposition que cet article ne visa pas à consacrer la liberté du commerce, mais plutôt celle de créer des associations essentiellement à but lucratif. On peut ensuite constater que la constitution du $1^{\mathrm{er}} / 08 / 1964$ rompit avec ce silence en précisant clairement en son article 44 que «l'exercice du commerce est garanti à tous les congolais sur le territoire de la République dans les conditions fixées par la loi nationale ». ${ }^{8}$

L'article 46 renchérit en disant : «que tout étranger qui se trouve sur le territoire de la République jouit de la protection accordées aux personnes et aux biens en vertu de la présente constitution, sauf, les exceptions établies par la loi nationale $\|^{9}$ or pour les étrangers, ils jouissaient des droits réservés aux congolais dans la mesure fixée par la loi nationale.

Bien plus la liberté du commerce et de l'industrie des congolais ont été reconnue d'une façon explicite par les constitutions congolaises. L'article 37,24 de la constitution du 17

4 L'article 5 de l'acte général de Berlin.

5 Idem.

6 NGUYEN C.T. et A1 : Lexique du droit des affaires zaïrois, Kin, 1979, P.79.

7 L'article 16 de la loi fondamentale du 30 Juin 1960.

8 L'article 44 de la Constitution RDC de 1964.

9 L'article 46 de la Constitution RDC de 1964. 
Juin 1967 et L'article 26 de la constitution révisée du 15 Août 1974 ainsi que la constitution du 15 Février 1978 précisent clairement que : »tous les congolais ont droit de constituer des associations et des sociétés $\gg{ }^{10}$

La constitution du 24 Juin 1967 à aborder la question dans son article 14, cette disposition qui garantissait les droits de propriété individuelle ou collective, la constitution mise à jour au $1^{\text {er }}$ Janvier 1983 la règlementer par son article $24 .{ }^{11}$

Le principe de la liberté de commerce et de l'industrie est le corolaire du principe de libre commerce pour qu'en application des thèses de l'économie libérale ou concertée, la loi de l'offre et de la demande apporte ses bienfaits spécifiques, sous la main invisible de la providence. La liberté fondamentale. Les pouvoirs publics ne peuvent sans prétexte d'assurer la police du commerce, interdire ou rendre pratiquement impossible l'exercice d'un commerce ou d'une industrie. En revanche l'exercice du commerce peut être règlementé dans l'intérêt général. Touchant à l'ordre public, la liberté des commerces ne peut être supprimée par de conventions particulières. Les restrictions légales sont néanmoins édictées dans des cas particuliers : par exemple l'interdiction d'exercer la profession commerciale peut être prononcée par les Tribunaux à titre des peines.

Le principe de la libre concurrence qui est d'application en droit congolais a pour objet d'empêcher que l'entreprise ne soit pas privée de la clientèle qu'elle s'est acquise ou celle qu'elle est susceptible d'attirer, par des moyens dont elle dispose. C'est pourquoi on a pu dire que c'est essentiellement le « droit à la clientèle » qui est protégé par des moyens font divers. ${ }^{12}$

La liberté ne peut s'exercer, sans égard aucun pour les droits et les intérêts légitimes des autres entreprises. La concurrence est parfois prohibée par l'effet de monopoles qui réservent l'une ou l'autre activité déterminée à certaines entreprises à titre exclusif. Mais les concurrents doivent surtout s'astreindre à respecter les règles de correction c'est-à-dire usage honnêtes du commerce dont la méconnaissance constitue la concurrence déloyale. ${ }^{13}$

A travers les lignes qui vont suivre, nous allons, tant que faire se peut, cerner les contours de la problématique des mécanismes de protection de la libre concurrence en République Démocratique du Congo et leur effectivité, l'exemple du Secteur des télécommunications. En adoptant la démarche méthodique suivante :

- D'abord analyser la structure du marché des télécommunications en droit congolais;

- La promotion de la concurrence;

10 L'article 24, 37 de la Constitution RDC de 1967 et 26 de la Constitution du 15/08/1974.

11 Depuis le mois d'Avril 1990, notre pays a amorcé des reformes des structures destinées à instituer un nouvel ordre constitutionnel et démocratique. C'est pourquoi la constitution du $1^{\text {er }}$ Janvier 1983 est remplacée par l'acte constitutionnel de la transition qui consacrait en terme semblables, la liberté de commerce.

12 Y VAN RYN et HEENER : Principes de droit commercial, Tome I n 185, P. 134 et 135.

13 MULOMBA M. : Cours de droit commercial général, G3 UNILU, 1999 - 2000, P.134. 
- Les procédés économiques qui portent atteinte à la concurrence et enfin les mécanismes de protection de la libre concurrence et leur effectivité, cas du secteur des télécommunications.

En étudiant la question de cette manière, nous espérons en donner une vue complète avant de faire des propositions de lège feranda spécialement au sujet de la loi nº13/2002 du 16 Octobre 2002 sur les télécommunication en République Démocratique du Congo et celle du 16/10/2002 portant création de l'autorité de régulation de la poste et des télécommunications qui en droit positif congolais organise semble-t-il les mécanismes des protections de la libre concurrence.

\section{ETUDE DES DIFFERENTES STRUCTURES DU MARCHE}

\section{LE MARCHE}

Le marché comprend plusieurs structures qui différent les unes des autres en fonction de la dimension et du nombre des opérateurs. Le marché est défini comme la rencontre d'un ensemble d'offres et des demandes d'un bien donnant lieu à échange sur base d'un prix, il s'agit du lien théorique ou se rencontre l'offre et la demande des produits ou de services qui sont considérés par les utilisateurs, par les acheteurs comme substituables entre eux et non substituables aux autres biens ou service offert. Au sens large on entend par marché l'ensemble des possibilités pour l'entreprise :

- en matière première (marché de l'achat)

- en main d'œuvre (marché du travail)

- en capitaux (marché de l'argent)

- en débouchés (marché de la vente).

$\mathrm{Au}$ sens usuel qui nous intéresse ici, on entend par marché uniquement celui de la vente, des débouchés. ${ }^{14}$

Dans ce sens, l'étude du marché peut s'analyser comme l'étude méthodique de la demande d'un produit et de l'offre qui y répond, en effet, le marché présuppose un comportement collectif des agents économiques à l'égard des biens offertes et demandés.

a) Le Monopole : nous rappelons ici qu'il y a monopole parfait chaque fois qu'un seul sujet à la disposition complète de l'offre et se trouve en face d'une pluralité d'acheteurs ${ }^{15}$ donc est une structure du marché caractérisée d'une seule production. Il peut résulter des facteurs naturels ou de la volonté de pouvoir public. L'existence d'un gisement unique ou d'une source d'eau minérale unique constitue le monopole naturel. Les activités d'assurance (SONAS) il s'agit du monopole légal.

b) L'OLIGOPOLE : cette nouvelle forme de représentation du marché s'apparente à la théorie de l'action humaine. Il s'agit de rechercher dans quelle mesure un sujet écono-

14 )VERHULT ALPHONSE P. : Economie d'entreprise, Kin, éd. Centre de Recherche Pédagogique, 1992.

15 H. GUITTON \& D. VITRY : Economie politique, éd. Dalloz, 1989. 
mique subit le prix du marché, ou au contraire impose aux autres son propre prix. On a constaté que cette action sur le prix était pourquoi la théorie activiste agissante ou stratégique du prix a été associée ou régime du petit nombre c'est-à-dire l'oligopole qui est ici considéré comme une structure du marché dans laquelle opère un nombre réduit d'opérateurs, entreprises dont les actions sont interdépendances. ${ }^{16}$

c) LE MONOPSONE : Dans le cas du monopsone, nous sommes en présence d'un acheteur unique qui se trouve en face d'une multitude de vendeur. Il concerne dans beaucoup des cas le marché des facteurs. Cependant, l'équilibre du monopsoneur s'obtient lorsque le coût marginal égal à la recette marginal et provoque les niveaux d'emploi et des rémunérations inférieures à ceux existant dans le marché de concurrence parfaite. ${ }^{17}$

d) CONCURRENCE : Elle est définie comme la structure du marché caractérisée par une confrontation libre d'un grand nombre d'offreurs et des demandeurs dans tous les domaines et pour tout bien et service matière première, travail, bien d'équipement et fonds prêtables. $^{18}$

\section{PROMOTION DE LA CONCURRENCE}

La concurrence est assurée par la répression des actes et comportements qui sont de nature à fausser son jeu et à restreindre la concurrence. Ces actes et comportements sont la concurrence déloyale, les pratiques restrictives de la concurrence, les ententes et abus des positions dominantes y compris les concentrations.

\section{a) La concurrence Déloyale}

L'ordonnance législative ${ }^{\circ} 41-63$ du 24 Février 1950 relatif à la répression de la concurrence déloyale stipule en son article 1 que : « lorsque, par un acte contraire aux usages honnêtes en matière commerciale ou industrielle, un commerçant, un producteur, un industriel ou un artisan porte atteinte ou crédit d'un concurrent, où lui enlève sa clientèle, ou d'une manière générale porte atteinte à sa capacité de concurrence, le Tribunal de première instance, sur poursuite des intéressés, ou de l'un d'eux, ordonne la cessation de cet acte ». ${ }^{19}$

L'Article 2 de l'ordonnance législative susmentionnée définie les actes constitutifs de la concurrence déloyale qui sont des actes qui créent ou tente de créer la confusion entre la personne, l'établissement ou les produits de l'auteur de ceci, l'établissement ou les produits d'un concurrent, les actes de dénigrement d'un concurrent.

L'étude de la jurisprudence fait ressortir 3 situations différentes dans lesquelles la déloyauté a été retenue.

16 SYSLOS LABINI : Oligopolise pnogreso technico, 1956.

17 H. GUITTON \& D. VITRY : Economie politique, éd. Dalloz, 1989.

18 CHAPIT YVES, Droit de la concurrence, collection que sais-je, P.U.F, Paris, 1991, P.125.

19 L'article 1 de l'Ordonnance Législative n41-63 du 24 Février 1950 relatif à la concurrence déloyale. 
- Le Dénigrement : il consiste à jeter le discrédit sur un concurrent en le critiquant dans l'intention de lui nuire. La preuve de la réalité des faits justifiant les critiques ne suffit pas d'exonérer l'acteur; les juges prennent en compte l'intention malveillante. ${ }^{20}$

- La confusion : Elle consiste à tenter de profiter la bonne renommée d'un commerçant en faisant croire à la clientèle qu'il s'agit de la même entreprise. Ladite confusion peut porter sur des produits imités sur le nom commercial, sur la marque, sur la disposition des locaux..$^{21}$ L'article 2 de l'ordonnance législative n ${ }^{\circ} 1-63$ du 24 Février 1950 relatif à la concurrence déloyale interdit les actes susceptibles de créer la confusion entre la personne, l'établissement et les produits des concurrences. ${ }^{22}$

La confusion est en quelque sorte l'inverse de dénigrement. Celui qui engendre la confusion cherche à s'approprier le succès de son concurrent client en trompant sa clientèle. ${ }^{23}$

- Désorganisation : Il s'agit d'essayer de désorganiser une entreprise rivale par différents moyens dont les principaux sont : - l'espionnage industriel.

- Le débouchage de personnel en vue de détourner la clientèle,

- Le détournement de commandes. ${ }^{24}$

Ces actes méritent d'être sanctionnés, il ne s'agit dont que des sanctions civiles.

b) Le Parasitisme : le parasitisme économique consiste à se placer dans le sillage d'autrui, à s'appuyer sur les efforts et les initiatives d'un opérateur économique, concurrent ou non pour conquérir une clientèle. ${ }^{25}$

Un arrêt du 21/01/1999 de la chambre commerciale à donné une définition du parasitisme économique : l'ensemble des comportements par lesquels un agent économique s'immisce dans le village d'un autre, afin de tirer profit, sans rien dépenser, de ses efforts et de son savoir.

La jurisprudence, dans un $1^{\text {er }}$ temps (année 1960, a jugé qu'il n'y avait pas concurrence déloyale, car de risque de confusion ce fut le cas pour MAZDA piles et automobiles, voiture et réfrigérateurs cas de la France.)

La jurisprudence récente condamne, même en l'absence de risque de confusion ou dénigrement, par exemple l'utilisation du slogan d'une autre entreprise non concurrente appropriation indue de plan. ${ }^{26}$

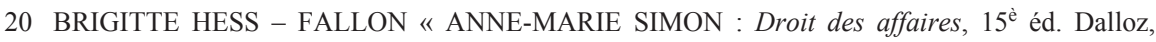
2003, P. 58-65.

21 Idem, P.352 S 3 è éd. L.G.D.J., P.67.

22 L'article 2 de l'ordonnance législative de 1950 relatif à la concurrence déloyale, $2^{2}$ éd. L.G.D.J., P. 352.

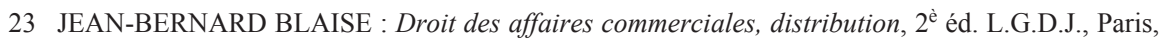
2000, P.352.

24 BRIGITTE HESS - FALLON « ANNE-MARIE SIMON : Op.cit, P.61.

25 G. RIPERT \& R. ROBLOT : Traité de droit commercial, L.G.D.J., $2^{\mathrm{e}}$ éd P.352.

26

BRIGITTE HESS - FALLON « ANNE-MARIE SIMON : Op.cit, P. 58-65. 
L'enjeu, à l'heure actuelle, consiste à transposer ce principe au niveau international et partant, à construire le cadre juridique d'une société globale en émergence dans la mouvance de l'ouverture des frontières et de la transnationalisation des pratiques économiques.

c) Coalition sur le prix

Elles sont des actions concertées dans le but de hausser ou de baisser le prix sur le marché, les producteurs peuvent se coaliser pour tirer un avantage quelconque sur le marché et une telle action est susceptible d'attenter à la libre concurrence. ${ }^{27}$

C'est pour cette raison que le législateur congolais à prohibé des coalitions sur le prix. L'article 15 du décret-loi du 20/03/1961 puni d'une servitude pénale de 15 jours à 3 ans et d'une amende de 10.000 à $300.000 \mathrm{FC}$ ou l'une de ces peines seulement. ${ }^{28}$

\section{d) Prix Imposé}

L'article 34 de l'ordonnance-loi de 1986 prohibe les faits pour toute personne d'imposer directement ou indirectement, un caractère minimal ou prix de revente d'un produit ou d'une prestation de service, ou à une marge commerciale. ${ }^{29}$

La libre détermination du prix de vente par le revendeur. Ce principe s'impose à toute personne, fournisseur, grossiste, importateur et vise tous les contrats, vente, prestation de service. Aussi en imposant les prix, les producteurs peuvent perturber le marché et modifier les structures économiques. Cette pratique est souvent d'application en matière de cession commerciale c'est-à-dire les chaînes de distribution. ${ }^{30}$

\section{e) Les ententes}

Elles sont un accord implicite ou explicite limité ou global entre deux ou plusieurs entreprises ou groupe d'entreprise qui tout en conservant leur personnalité juridique harmonisent leur politique pour réduire la concurrence dans une branche d'activité. Elles se réalisent sous forme de cartel, des coalitions, des pools, consortium et peut porter sur le prix, les conditions de vente, le volume de production et la nature de la production. ${ }^{31}$

Les ententes sur le prix entretiennent la hausse ou la baisse anormale de prix. L'entrave aux progrès techniques est un refus concerté des producteurs d'utiliser une technique nouvelle dans le but d'amortir leurs équipements ou de préserver leurs parts des marchés.

27 MARIE MALORI VIENALE : Op.cit, P. 227-278.

28 L'article 15 du décret-loi du 20/03/1961 tel que modifié par l'ord. Loi n83/026 du 12/09/1983portant régime de prix.

29 L'article 35 de l'Ordonnance loi. De 1981 relatif à la réglementation de prix.

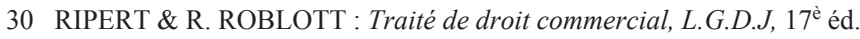

31 TSHIZANGA MUTSHIPUNGU : Cours de droit de la concurrence, L1 Droit UNILU, Département de droit économique et social, 2008. 


\section{f) La concentration}

Elle consiste à exécuter dans une même entreprise ou dans un groupe d'entreprises ayant des intérêts communs des opérations connexes que la spécialisation aurait réparties entre plusieurs entreprises indépendantes. ${ }^{32}$

\section{g) Incidence de la Concentration sur la Concurrence}

La concentration peut par la coordination de comportement concurrentiel des entreprises indépendantes entrainer l'élimination de la concurrence en réduisant l'intensité et la rivalité concurrentielle par la position dominante qu'elle crée. Elle est également susceptible d'entraver la concurrence dans la mesure où les entreprises non intégrées peuvent être des avantagées en acquisition des matières première (IN PUT).

Elles peuvent être disséminées au niveau de la qualité ou de la régularité de l'approvisionnement de service.

\section{LES MECANISMES DE PROTECTION DE LA LIBRE CONCURRENCE EN R.D.C. ET LEUR EFFECTIVITE CAS DU SECTEUR DES TELECOMMUNICATION}

Les mécanismes de protection de la libre concurrence en République Démocratique du Congo et leur effectivité sont prévus par les différents textes légaux ci-après :

1) Loi $n^{\circ} 011 / 2002$ sur la poste;

2) Loi-cadre $n^{\circ} 013 / 2002$ sur les télécommunications en RDC;

3) Loi ${ }^{\circ} 014 / 2002$ portant création de l'autorité de régulation de la poste et des télécommunications;

4) L'Arrêté Interministériel $n^{\circ} \mathrm{CAB} / \mathrm{MIN} / \mathrm{PTT} / 030 / \mathrm{MM} / 2002$ et $\mathrm{N}^{\circ} \mathrm{CAB} / \mathrm{MIN} / \mathrm{ECO}-\mathrm{FIN}$ \& BUD/104/2002 portant fixation du coût d'interconnexion locale et internationale;

5) Arrêté Interministériel $n^{\circ} 006 / \mathrm{CAB} / \mathrm{MIN} \& \mathrm{BUD} / 2003$ et $n^{\circ} 001 / \mathrm{CAB} / \mathrm{MIN} / \mathrm{PTT} /-2003$ portant fixation de la taxe terminale sur les communications internationales entrantes;

6) Arrêté Ministériel $\mathrm{n}^{\circ} \mathrm{CAB} / \mathrm{MIN} / \mathrm{PTT} / 0027 / 31 / 93$ fixant les conditions d'exercice des activités dans le secteur des télécommunications.

7) Arrêté Département $n^{\circ} \mathrm{DENI} / \mathrm{CAB} / 06 / 013 / 87$ du 26/05/1987 fixe les modalités de la saisine de la concurrence.

1. Loi ${ }^{\circ} 012 / 2002$ du 16 Octobre 2002 sur la poste

La loi qui régit le secteur postal à ce jour date de 1968, année de la publication de l'ordonnance-loi no68-045 du 20 Janvier 1968 sur le service postal. Depuis lors, cette loi n’a subis aucune modification, malgré l'évolution rapide de l'environnement des technologies pos-

32 Jacques MIN ALEXIS \& SCRANHE GUY : Droit économique, Collection que sais-je, P.U.F, Paris, 1989. 
tales. Par cette loi le législateur congolais cherche à mettre à la disposition de notre pays un instrument juridique définissant de matière plus claire les principes, les règles et es institutions devant régir les activités et les services de postes. ${ }^{33}$

A la suite de la libération des transports et des communications ou plan mondial, notre cher et beau pays à introduit des reformes dans le secteur de postes. Celles-ci dit porté principalement sur la dissociation des activités du secteur postal de celles des télécommunications, sur la limitation du monopole, la séparation des fonctions de régulation de l'exploitation et sur l'obligation de fournir le service universel. Ces reformes ont eu des effets bénéfiques pour le pays qui les ont initiées, notamment :

- L'augmentation de la couverture postale, l'amélioration de la qualité de service, l'accroissement de la contribution du secteur du budget de l'Etat.

La République Démocratique du Congo ne peut se tenir en manque du courant mondial, d'autant plus que sur le territoire national, l'on enregistré l'émergence des opérateurs privés nationaux et internationaux, formels et informels qui, de fait, dit ont ouvert le marché concurrentiel. Par ailleurs, le secteur postal connait une grande mutation consécutive à l'évolution des techniques de la poste aux lettres et de la poste financière. C'est cette évolution qui a rendu nécessaire la révision et l'adaptation du cadre légal et réglementaire régissant le secteur suivant la nouvelle politique postale du gouvernement. ${ }^{34}$

La politique du gouvernement de l'époque s'est fixée les principaux axes tendant à :

- Limiter le monopole à la fourniture du service universel et veiller à permettre à l'exploitant qui en a la charge à générer des revenus suffisants pour accéder aux nouvelles technologies et couvrir les frais inhérents à la fourniture des services universels.

- Ouvrir le marché à la concurrence en vue de fournir les services à valeur ajoutée de meilleure qualité et les services nouveaux (participation de privé ou développement. Du service postal dans un environnement de la concurrence loyale).

- Etendre la couverture postale nationale par une plus grande prise en compte des zones rurales et isolées. ${ }^{35}$

L'exposé chronologique renseigne que depuis l'Etats indépendant du Congo en passant par la période coloniale, aussi bien qu'après l'indépendance sous l'empire de l'ordon-nance-loi $\mathrm{n}^{\circ} 68-045$ du 20 Janvier 1968 sur le service postal comme sous celle de l'or-donnance-loi $\mathrm{n}$ ${ }^{\circ} 68-475$ du 13 Décembre .... Portant création de l'OCPT. Le service postal a toujours été considéré comme un service public c'est-à-dire un besoin de la collectivité que les pouvoirs publics se doivent satisfaire à sa population en recourant aux règles de gestion du droit administratif.

Jusque-là le service public, entendu dans ce sens, justifiait le monopole exercé par l'Etat en ce qu'il légiférait, réglementait et exploitait les services des postes. Mais, la tendance à la libération des activités économiques constatée par le monopole, a entrainé le sec-

33 Exposé des motifs de la loi nº12/2002 du 16 Octobre 2002 sur la poste.

34 Idem.

35 Journal officiel numéro spécial - 25 Janvier 2003 poste et télécommunication, pages 4 et 5. 
teur postal à limiter l'étendue du monopole et à couvrir le marché à la concurrence des opérateurs privés. ${ }^{36}$ Cette constatation a été confirmée par la Résolution C 91 du programme général d'action de WASHINGTON, (PGAW) qui recommande - l'autonomie de gestion, l'assouplissement et l'adaptation du monopole; la réservation de certains services ou monopole. ${ }^{37}$

Par ailleurs, la Résolution C 95/1994 portant sur la stratégie postale de Séoul a abordé dans le même sens, en recommandant de :

- définir l'étendue du monopole en tenant compte des ressources nécessaires pour assumer une balance commerciale solide de la poste qui a une obligation du service public sur tout le territoire national.

- Instaurer un système de contrôle de l'application de la réglementation relative ou monopole

- Définir les limites de la concurrence loyale et équitable dans les services internationaux et nationaux en se conformant aux obligations découlant des accords de GIAIT. ${ }^{38}$

\section{Loi - Cadre n013/2002 du 16 Octobre 2002 sur les Télécommunications en R.D.C}

Cette loi Cadre sur les télécommunications dote le pays d'un instrument juridique définissant de manière précise les principes, les institutions qui régissent les activités, les réseaux et les services de télécommunications.

L'évolution technologique est l'un des facteurs fondamentaux de toutes les invitations du secteur de télécommunication et des adaptations qu'il exige. ${ }^{39}$

Au plan économique, les télécommunications, à la suite de l'expansion des services qui permettent l'évolution technologique, deviennent le système nerveux des économies modernes et leur bonne ou mauvaise gestion détermine la capacité d'un Etat à assurer les attributions de sa souveraineté. La République Démocratique du Congo ne pouvait plus longtemps encore se tenir à l'écart de la marche du monde vers la troisième révolution, celle de l'information sous peine de pénaliser sa population par des communications insuffisantes en qualité et en qualité et limitées par sa participation au marché mondial. ${ }^{40}$

La politique des télécommunications de la loi susmentionnée fixe, les principaux axes qui l'articulent autour des recommandations suivantes :

- permettre l'accès de l'ensemble de la population aux services de base du téléphone et du télex en zone rurales et urbaines grâce à un goût raisonnable;

36 J.O. Numéro Spécial - 25 Janvier 2003 - Poste et Télécommunication, page 5.

37 La Résolution C 91 du programme Général d'action de Washington.

38 La Résolution C 95 de la stratégie postale de Séoul.

39 Exposé des motifs de la Loi-cadre n013/2002 du 16 Octobre 2002 sur les télécommunications en République Démocratique Congo.

40 Journal Official - Numéro Spécial -25 Janvier 2003 poste et télécommunication, P.18. 
- Favoriser l'introduction de nouvelle technologie pour répondre aux besoins dans cesse croissant et en mutation rapide des milieux d'affaires.

- Libéraliser les services à valeur ajoutée, les services nouveaux, les équipements terminaux pour susciter leur accroissement en nombre, la concurrence et la compétitivité et les introduire également en zone rurales, favoriser l'interconnexion des réseaux et des services au niveau national, régional et international.

- Réduire le champ du monopole en soumettant à la concurrence les services de base, ceux à valeur ajoutée ainsi que les services nouveaux..$^{41}$

Il est important de rappeler ici que la loi susdite à introduite des innovations dans le secteur des télécommunications. Cette innovation concerne :

- Les attributions du ministre en charge des télécommunications, sans préjudice des dispositions des autres textes légaux ou réglementaires pris par ailleurs dans le même domaine;

- La création d'une autorité de régulation, placée sous la tutelle du ministre des postes et des télécommunications. Cette création consacre le principe de l'indépendance des fonctions de régulation de celles d'exploitation.

- La participation du secteur privé au développement de télécommunication dans un environnement concurrentiel loyal et la philosophie de base qui sous-entend la loi se résume en un réaménagement du régime de monopole par l'introduction de règles de concurrence pour les segments du marché ouvert à la concurrence.

Le monopole défini par l'ordonnance législative n²53/Télécommunication du 23 Août 1940 était fondé sur le caractère de service public de télécommunication dans notre pays, parce que celle-ci entendue comme un besoin collectif que les pouvoirs publics se devaient satisfaire à l'aide de technique et méthodes relatives à la gestion publique assujettie aux règles de droit public..$^{42}$

Par ailleurs, l'Etat a, lui-même, directement géré les télécommunications par le biais de l'administration publique, jusqu'en 1968, par celui d'un organisme décentralisé, l'Office Congolais des Postes Télécommunications « O.C.P.T»; depuis 1968 ou indirectement et de manière supplétive par les personnes morales bénéficiaires de concession ou l'autorisation particulière ayant conclu avec l'Etat des conventions en vue de l'intervention de leurs installations dans les services publics de télécommunications.

Il a fallu donc, réaménager le monopole sans toute fois libéraliser totalement le marché de télécommunication. Ce monopole est cependant, tempéré par la faculté laissé au Ministre des Télécommunications d'autoriser, à titre exceptionnel et aux conditions fixées par la loi, un exploitant concessionnaire de service public des télécommunications à disposer de ses propres voies de sortie à l'international (article 38, alinéa 3). ${ }^{43}$

41 Idem.

42 Ordonnance législative n²53/Télécommunication du 23 Août 1940.

43 L'Article 11 et 38 de la loi cadre $n^{\circ} 013 / 2002$ sur les télécommunications en République Démocratique du congo.. 
L'ouverture à la concurrence s'applique aussi aux services de base qu'aux services à valeur ajoutée et aux services nouveaux. De même, l'importation et la fabrication des équipements et des terminaux sont libéralisées.

Le réaménagement du monopole et l'introduction de la concurrence à conduit à une profonde restructuration du secteur des télécommunications par séparation des fonctions de réglementation et d'exploitation.

En effet, les mécanismes de protection de la libre concurrence et leur effectivité dans le secteur de télécommunication en droit congolais a été abondé ou analysé par la loi sous examen dans son article 1 qui dispose ce qui suis : « la présente loi régit le secteur de télécommunication en République Démocratique Congo a pour objet de : - fixer les modalités de détentions d'installations et d'exploitation de télécommunication sur toute l'étendue du territoire nationale;

- Garantir le développement harmonieux et intégré des réseaux et services de télécommunications

- Faciliter la mobilisation des ressources financières par la participation du secteur privé ou développement de télécommunication dans un environnement concurrentiel loyal. ${ }^{44}$

3. Loi n $014 / 2002$ du 16 Octobre 2002 portant création de l'autorité de régulation de la Poste et des Télécommunications en R.D.C

Cette loi a pour objet la création d'un Organe de régulation, du secteur des postes et télécommunications dénommé AUTORITE DE REGULATION DE LA POSTE DES TELECOMMUNICATIONS DU CONGO en sigle A.R.P.T.C.

Elle sert à créer un environnement acceptable et d'harmoniser les rapports entre les différents intervenants dans les secteurs des postes et télécommunications. Il ressort que l'absence d'une autorité de régulation autonome vis-à-vis de pouvoirs publics occasionne une exploitation anarchique du secteur des postes et télécommunication. ${ }^{45}$

Il sied de souligner ici que le statut de l'autorité de régulation a une certaine particularité. Il est du reste observer que la plupart des modèles expérimentés avec succès dans plusieurs pays sont revêtus de statut autonome, ceci étant une conséquence logique de l'ouverture du secteur à la concurrence. L'autorité de régulation a pour mission de :

- Veiller au respect des lois, règlements et conventions en matière des postes et télécommunications.

- Définir les principes d'interconnexion et de tarification des services publics des postes et télécommunications;

44 L'article 1 de la loi cadre $n^{\circ} 013 / 2002$ du 16 Octobre 2002 sur les télécommunications en République Démocratique Congo.

45 L'article 1 de la loi cadre $n^{\circ} 014 / 2002$ du 16 Octobre 2002 sur les télécommunications en République Démocratique du Congo. 
- Elaborer et gérer le plan national de numérotation; ${ }^{46}$

- Suggérer toute modification législation ou règlementaire qui lui paraissent nécessaire à l'évolution des secteurs de postes et télécommunications et ou développement de la concurrence.

- Protéger sur le marché des postes et télécommunications, les intérêts des consommateurs et des opérateurs en veillant à l'existence et à la promotion d'une concurrence effective et loyale et prendre toutes les mesures nécessaire à l'effet de rétablir la concurrence ou profit des consommateurs; etc. ${ }^{47}$ L'autorité de régulation est constituée d'un collège et d'une administration. Le collège de l'autorité de régulation est composé de sept membres à savoir, un président, un vice-président et cinq conseillers. Dont le président et son vice sont nommés par le Président de la République.

Les autres membres du collège de l'autorité de régulation sont nommés par le président de la République dont deux sur proposition du parlement et trois sur proposition du ministre ayant les postes et les télécommunications dans les attributions. ${ }^{48}$

S'agissant du personnel, elle emploie trois types de personnel :

a) Le personnel recruté conformément à la loi n $152 / 2002$ du 16/10/2002 portant code du travail congolais.

b) Le personnel de carrière des services publics de l'Etat en position de détachement.

c) Les agents en provenance des entreprises publiques. ${ }^{49}$

L'Autorité de régulation dispose quant aux finances, les ressources ordinaires, provenant des revenus et des prestations, la taxe de rémunération, celle de régulation, taxe parafiscales autorisée par la loi financière, les frais d'administration lié à l'étude du dossier d'octroi ou de renouvellement des licences d'une part d'autre part les ressources extra ordinaire comprennent : les avances remboursable du trésor, d'organisation publique, les subvention dons, legs et toute autre recettes en rapport avec son activité. ${ }^{50}$

Pour ce qui est du régime fiscal, il est important de préciser ici que l'Autorité de régulation de poste et des télécommunications est exemptée de tous impôts, taxes et redevances. ${ }^{51}$

46 L'article 3 de la n ${ }^{\circ} 14 / 2002$ du 16 Oct. 2002 portant création de l'autorité de régulation de la poste et des télécommunications.

47

48 L'article 9 à 10 de loi n $14 / 2002$ du 16 Octobre 2002 portant création de l'Autorité de régulation de la poste et des télécommunications p.10.

49 L'Article 19 de la loi N"14/2OO2 du 16 octobre2002 Portant création de l'autorité de régulation de la poste et des télécommunications. p.19.

50 L'article 21 de la loi n ${ }^{\circ} 14 / 2002$ du 16 Octobre 2002 portant création de l'Autorité de régulation de régulation de la poste et des télécommunications.

51 L'article 25 de la loi n⿳亠丷厂 $14 / 2002$ du 16 Octobre 2002 portant création de l’Autorité de régulation de régulation de la poste et des télécommunications. 
Sa gestion comptable est assurée par le président du collège de l'autorité de régulation qui est l'ordonnateur du budget en recette qu'en dépenses. ${ }^{52}$

La dissolution de l'autorité de régulation peut être décidée par le président de la république qui en désigne le liquidateur. ${ }^{53}$

Au-delà de différentes dispositions légales analysées précédemment qui garantissent les mécanismes de protection de la libre concurrence et leurs effectivités des sociétés des télécommunications en droit congolais.

Le législateur congolais soucieux de protéger et promouvoir, se secteur a encore une fois institué un organisme dénommée "LA COMMISSION DE LA CONCURRENCE » par un arrêté départemental n ${ }^{\circ} \mathrm{DENI} / \mathrm{CAB} / 06 / 013 / 87$ du 26/05/1987. L'article 8 de l'arrêté susvisé fixe les modalités de saisine de ladite commission. Il dispose ce qui suit « la Commission de la concurrence se saisit d'office de tout dossier relevant de sa compétence. Elle également saisie de toute requête des consommateurs, des concurrents ou de toute personne physique ou morale intéressée par la concurrence $» .{ }^{54}$

Lorsque cette commission est régulièrement saisie, elle fait examiner le dossier par l'une de ses trois sections. Elle peut mener des enquêtes appropriées sur le terrain dûment autorisé par le ministre de l'économie nationale et de l'industrie. Cette section dépose ses conclusions aux membres de la commission pour des observations éventuelles. En cas des présomptions de faute retenues à charge d'un opérateur économique, qui a fait usage de pratique anticoncurrentielle, une convocation signée par le ministre ou son délégué est adressé à ce dernier, et un délai de 8 jours francs est accordé dès la réception de la convocation pour comparaitre devant la commission.

Sous réserve de l'article 5 du présent arrêté, la commission procède à l'audition des opérateurs économiques, incriminés au cabinet du ministre de l'économie nationale et industrie.

Le président est tenu de porter à la connaissance de l'agent économique les faits qui lui sont reprochés au regard des lois et de règlements en la matière. Ces derniers peuvent présenter ses moyens des défenses. Après l'audition des intéressés, la commission établie un procès-verbal d'audition signé par l'agent économique intérimaire ou son représentant avec les membres de la commission qui jouent le rôle d'officier de police judiciaire.

Le taux de l'amende transactionnelle est fixée par la commission dans les limites des dispositions légales en la matière et l'opérateur économique régulièrement sanctionné doit s'acquitter de bonne foi de l'amende à laquelle il a été condamnée.

En cas de non paiement le dossier sera transféré ou ministre de la justice.

52 L'article 26 de la loi n $14 / 2002$ du 16 Octobre 2002 portant création de l'Autorité de régulation de régulation de la poste et des télécommunications.

53 L'article 29 de la loi n ${ }^{\circ} 14 / 2002$ du 16 Octobre 2002 portant création de l'Autorité de régulation de régulation de la poste et des télécommunications.

54 L'article 8 de l'Arrêté Départemental nDENI/CAB/06/03/87 DU 26/05/1987 portant Commission de la concurrence. 
Tandis qu'en Belgique, il n'y a que deux organes tous compétent pour résoudre ou régler le cas d'abus de concurrence à savoir : - le conseil de la concurrence.

Direction Générale de la concurrence et la répression des fraudes, ont pour mission les enquêtes et poursuites.

\section{CONCLUSION}

La concurrence et la libéralisation des échanges ont globalement le même objectif : réduire ou supprimer les obstacles et les distorsions affectant les marchés. Le principe général qui doit, aux yeux de certains économistes, guider la définition des règles, est celui du maintien d'une concurrence sur des marchés contestables. Ce principe doit s'appliquer indistinctement aux entreprises comme aux Etats

Le Développement de la puissance économique des entreprises comporte de nombreux risque d'abus. D'une part, il s'accompagne parfois d'une entrave ou jeu normal de la concurrence. D'autre part, il n'est pas rare qu'il entraîne une dégradation du sort des consommateurs. La normalisation de la compétition concurrentielle s'impose pour sauvegarder le principe de la libre concurrence entre les différentes sociétés œuvrant dans le secteur de la télécommunication, pour promouvoir le développement économique du pays. Le libre jeu de la concurrence est la meilleure façon d'orienter la vie économique, car certains économistes libéraux adoptent l'analyse d'Adam Smith de la main invisible qui régule le marché. Pour que la concurrence soit efficace, elle doit être réglée et doit suivre certaines normes. Les excès de liberté n'entravent pas le fonctionnement d'une économie véritable du marché. En République Démocratique du Congo, l'opérateur économique victime des pratiques anticoncurrentielles, dispose de deux actions :

- Une action spécifique c'est-à-dire une action de cessation d'un acte anticoncurrentiel appelé également une concurrence déloyale prévu par l'ordonnance législative n $41 / 63 \mathrm{du}$ 24 Février 1950;

- Et une action de droit commun c'est-à-dire une action en Responsabilité civile conformément à la disposition de l'article 258 du Code Civil Congolais Livre Troisième.

Il est vrai que le système économique congolais se fonde sur la liberté du commerce et l'industrie qu'implique la libre concurrence, raison pour laquelle, une protection de cette liberté pour les sociétés des télécommunications a été matérialité par la mise sur pied, par le législateur congolais d'une série de réglementation idoines citée ci-haut.

Il sied de rappeler ici que les mécanismes de protection de la libre concurrence par les sociétés de télécommunication en droit congolais sont observés. Mais dans la vie pratique, il y a quelque déviation constaté par exemple la société AFRICELL-RDC, s'est vue couper le 08 novembre 2012 l'interconnexion par la société AIRTEL-CONGO, VODACOM et TIGO lui a infligé vingt quatre heures plus tard le même traitement et sans aucune explication, sans respecter même le contrat d'interconnexion qui fixe les conditions de résiliation au de suspension dudit contrat. 
Certaine spéculations explique que les trois entreprises citées ci-haut ont réagi ainsi pour montrer leur désaccord quant au FREE-RESEAU (un privilège accordé au nouveau venu dans le monde de télécommunication ou une connexion non payante accordée à AFRICELL-RDC. Cette dernière s'en est remise à l'autorité compétente qui se trouve être l'autorité de régulation de poste de télécommunication qui les a rappelé à l'ordre en soutenant que « tout réseau de télécommunication établit en République Démocratique du Congo est obligé de s'interconnectés...

Et les responsables de la société AFRICELL-RDC ont menacé d'aller en justice contre AIRTEL, VODACOM et TIGO, au cas où ces dernières récidivent.

Pour clore, nous disons que tous ses différents textes légaux et les institutions susvisées sont destinés à garantir la liberté de la concurrence par les opérateurs de télécommunication de différents réseaux. Ils ont le droit à la libre confrontation de leurs activités économiques.

Tout comportement d'une ou plusieurs entreprises tendant, par l'abus d'une position dominante limite l'accès des marchés concurrentiel, restreindre indûment la concurrence, ou qui risquerai d'avoir les effets préjudiciels pour les consommateurs (les ententes, le parasitisme économique, le refus de vente, coalition sur le prix, le prix imposés, la concentration d'entreprise, devraient être strictement interdit : voir même ériger ce comportement en infraction :

- Les entreprises ou sociétés des télécommunications en positions dominantes, jugée indispensables pour la réalisation du progrès économiques et social devrait en principe faire l'objet de surveillante particulière;

- Il va falloir à ce que le législateur congolais mettent sur pied des techniques juridique de protections de consommateurs et les opérateurs des télécommunications pour une éducation qui occuperait sans doute une place de choix, cette éducation aurait pour finalité de former les principaux acteurs pré rappeler à la dynamique, à la vigilance, à l'honnête, loyauté à l'égard des autres concurrents pour un élan nouveau.

- Un élan favorable à l'assainissement de la vie des affaires;

- Les dispositifs législatifs relatifs aux sociétés des télécommunications doivent s'accompagnés inévitablement d'une de conscience collective et d'une mise aux point de technique juridique favorables aux intérêts des consommateurs.

Enfin de compte, on peut observer que la politique commerciale partagent un objectif complémentaire fondamental, l'élimination et la réduction des barrières aux marchés et les distorsions de ceux-ci. Lorsque les objectifs communs sont poursuivis avec vigueur, la libéralisation des échanges et l'application de la politique de concurrence sont complémentaires et se renforcent mutuellement. 
Il y a de la place pour tout le monde dans le marché de télécommunication en République Démocratique du Congo. Nous demandons aux concurrents de jouer franc jeu.

\section{BIBLIOGRAPHIE}

\section{TEXTES DES LOIS}

1. L'acte général de Berlin du 26 Février 1985

2. La loi fondamentale du $3 \mathrm{O}$ juin 1960

3. La constitution de la République Démocratique du Congo de 1964

4. La constitution de la République Démocratique du Congo de 1967

5. La constitution de la République Démocratique du Congo de 1974.

6. La constitution de la République Démocratique du Congo du 28 février 2006.

7. Le décret du 30 janvier 1940 mis à jour au 31 mais 1982 portant code pénal zaïrois.

8. Ordonnance législative $\mathrm{N}^{*} 41-63$ du 24 - février 1950 relative à la concurrence déloyale.

9. La loi N"012/2002 du 16 octobre 2002 sur la poste.

10. Loi- cadre N"13/2OO2 sur les télécommunications en République Démocratique du Congo.

11. Loi N“014/2002 portant création de l'autorité de régulation de la poste et des télécommunications.

12. Arrêté interministériel N"CAB/MIN/PTT O3O/MM 2002 et CAB/MIN ECO.FIN et BUDGET/1O4/2002 Portant fixation du coût d'interconnexion locale et internationale.

13. Arrêté Ministériel N"CAB/MIN/PTT/0027/31/1993 Fixant les conditions d'exercice des activités dans le secteur des télécommunications.

14. Arrêté Départemental N“DENI/CAB/O6/O3/87 du 26/05/1987 portant commission de la concurrence

II. OUVRAGES :

1. LUKOMBE NGHENDA : Droit congolais des sociétés, tome 1 P.U.C 1999.

2. ALI CENK KESKIN, Pour un nouveau droit international de la concurrence, Harmattan, Paris 2009

3. DEBROCK Christian/Brunelle Dorval, « Globalisation et nouveaux cadres normatifs ", cahiers de recherche-CELM (98-02), Groupe de recherche sur l'intégration continentale (GRIC), Université du Québec à Montréal, septembre 1998, p. 15.

4. NGUYEN C.T et A1 : Lexique du droit des affaires zaïrois, Kinshasa, 1979.P79.

5. JVAN RYN et HEENER : Principes de droit commercial T.I N"185.

6. VERHULT ALPHONSE P : Economie d'entreprise, Kinshasa, éd centre de recherche pédagogique. 1992.

7. H GUITON et D. VITRY : Economie politique, éd Dalloz 1989.

8. SYSLOS-LABINI : Oligopolise progreso technico, 1956. 
9. CHAPIT YVES : Droit de la concurrence, collection que sais-je P.U.F, Paris 1991.P125.

10. CHRISTIANT VAN LIERD : Economie politique kin C.P.R 1988.P27.

11. BRIGITTE HESS-FALLON et ANNE-MARIS SIMON : Droit des affaires, $15^{\text {ème }}$ éd DALLOZ, 2003.P 61-62.

12. JEAN-BERNARD BLAISE : Droit des affaires les commerçants; distribution, 2 ème éd L.G.D.J.Paris 2000; p.352.

13. MARIE MALORI VIRGNALE : Droit de la concurrence $2^{\text {ème }}$ éd armond collin paris $2 \mathrm{OO} 3$ p. 270.

14. G. RIPERT $\infty$ ROBLOTT : Traité de droit commercial, L.G.D.J,17 er éd.

15. JACQUEMIN ALEXIœSCRANCHE GUY : Droit économique collection que sais-je P.U.F, paris 1989. 